\title{
EQUIVALENCE OF LAGRANGIAN GERMS IN THE PRESENCE OF A SURFACE
}

\author{
WOJCIECH DOMITRZ AND STANISŁAW JANECZKO \\ Institute of Mathematics, Warsaw University of Technology \\ Pl. Politechniki 1, 00-661 Warszawa, Poland \\ E-mail:wdomitrz@ipe.pw.edu.pl, janeczko@alpha.im.pw.edu.pl
}

\section{Dedicated to Professor Stanistaw Eojasiewicz for his 70th birthday}

1. Introduction. Let $\omega$ be a closed two-form on a smooth manifold $M$. Most of interesting singular symplectic structures on $M$ are given in the form of pull-back $\omega=\phi^{\star} \omega_{0}$ by a smooth map $\phi: M \rightarrow R^{2 n}$ from a symplectic structure $\omega_{0}$ on $R^{2 n}[7,9,5,6]$. The natural structure group of such singular symplectic manifolds is defined by integration of $\phi$-liftable Hamiltonian vector fields on $\left(R^{2 n}, \omega_{0}\right)$. Action of this group on the space of Lagrangian varieties in $\left(R^{2 n}, \omega_{0}\right)$ provides the classification of maximal isotropic varieties in pulled-back singular symplectic structure. Hence there is a natural question to ask: How does the local intersection data determine the orbits of the group of symplectomorphisms preserving singular values of $\phi$ and acting on the space of Lagrangian germs? This question may be reformulated as a generalization of the classical problem: Under which conditions the two Lagrangian germs are equivalent? In this paper we attempt to answer this question imposing the genericity conditions on the classified symplectic objects and assuming the set of critical values of $\phi$ is a smooth surface in $R^{2 n}$. Another motivation to our study comes from the theory of symplectic triads $[1,10]$ and its generalization. However the local invariants considered in this paper do appear as the classified not necessary typical common positions of the considered two surfaces. Our aim is to construct all these invariants through the technique of finding the corresponding local normal forms. In Section 2 we classify the germs of generic pairs $(H, L ; p)$, where $H$ is a hypersurface in $R^{2 n}$ and $L$ is a Lagrangian submanifold in $\left(R^{2 n}, \omega_{0}\right)$. Then in Section 3 we consider the slightly different case if $H$ is any coisotropic submanifold of $\left(R^{2 n}, \omega_{0}\right)$. It is known that even-dimensional submanifolds in general position in the symplectic space

1991 Mathematics Subject Classification: Primary 57R45; Secondary 53B21.

Research of the authors supported by KBN grant 3P03A06910.

The paper is in final form and no version of it will be published elsewhere. 
are symplectic. In Section 4 we find the normal forms of pairs $(H, L ; p)$ provided $H$ is symplectic and $H$ and $L$ are in the transversal and nontransversal positions.

2. $H$-relative Lagrangian submanifolds. Let $\left(M, \omega_{0}\right)$ be a symplectic manifold, $\omega_{0}$ be in the Darboux form. Let $H \subset M$ be a smooth hypersurface in $M$. By $(H ; p)$ we denote the germ of $H$ at $p$.

Definition 1. A pair of germs $(H, L ; p)$, where $(L ; p)$ is a germ of a Lagrangian submanifold, is called an $H$-relative Lagrangian submanifold of $M$.

Theorem 1. If $L$ is transversal to $H$ at $p \in L \cap H$ then the pair $(H, L ; p)$ is symplectically equivalent to the following one

$$
\left(\left\{(x, y) \in R^{2 n}: x_{1}=0\right\},\left\{(x, y) \in R^{2 n}: y_{i}=0 \text { for } i=1, \ldots, n\right\} ; 0\right) .
$$

Any two $H$-relative Lagrangian submanifolds which are transversal to $H$ are $H$-relative symplectically equivalent, i.e. there exists a symplectomorphism $\Phi$ which preserves a hypersurface $H$ and maps one Lagrangian manifold into the other.

Proof. It is easy to see that $H$ is symplectically equivalent to

$$
\left\{(x, y) \in R^{2 n}: x_{1}=0\right\} .
$$

$L$ is $H$-relative symplectically equivalent to a Lagrangian submanifold generated by a function

$$
S: R^{n} \ni x \mapsto S(x) \in R,
$$

because $L$ is transversal to $H$. A symplectomorphism

$$
\phi(x, y)=(x, y-\operatorname{grad} S)
$$

maps $L$ into $\left(\left\{(x, y) \in R^{2 n}: y_{i}=0\right.\right.$ for $\left.\left.i=1, \ldots, n\right\} ; 0\right)$.

It is easy to prove the following lemma.

Lemma 2. If $L$ is not transversal to $H$ at $p \in H \cap L$ then the pair $(H, L ; p)$ is symplectically equivalent to

$$
\begin{aligned}
\left(H_{0}, L_{0} ; 0\right)=\left(\left\{(x, y) \in R^{2 n}: x_{1}\right.\right. & =0\}, \\
& \left.\left\{(x, y) \in R^{2 n}: x_{i}=-\partial S / \partial y_{i} \text { for } i=1, \ldots, n\right\} ; 0\right)
\end{aligned}
$$

where $S$ is a function-germ such that

$$
\left.d\left(\partial S / \partial y_{1}\right)\right|_{0}=0 .
$$

Proof. It is clear that $H$ is symplectically equivalent to $H_{0}=\left\{(x, y): x_{1}=0\right\}$ and $L$ may be simultaneously turned into

$$
L_{0}=\left\{(x, y): x_{I}=-\partial S / \partial y_{I}\left(y_{I}, x_{J}\right), y_{J}=\partial S / \partial x_{J}\left(y_{I}, x_{J}\right)\right\},
$$

where $I \cup J=\{1, \ldots, n\}, I \cap J=\emptyset, 1 \in I([3])$. Then by the symplectomorphic change of variables

$$
\Psi\left(x_{I}, x_{J}, y_{I}, y_{J}\right)=\left(x_{I},-y_{J}, y_{I}, x_{J}\right),
$$

we get the desired formula. 
Now we need the group $G_{H}$ of germs of symplectomorphisms which preserve the fibration $(x, y) \rightarrow y$ and the hypersurface $H=\left\{(x, y) \in R^{2 n}: x_{1}=0\right\}$ for the representation of $(H, L ; p)$. Every element $\Phi$ of this group can be defined as a lifting of a diffeomorphism $\phi: R^{n} \ni y \mapsto \phi(y) \in R^{n}$ which preserve the fibration over $\left(y_{2}, \ldots, y_{n}\right)$, i.e. $y=\left(y_{1}, y_{2}, \ldots, y_{n}\right) \mapsto \bar{y}=\left(y_{2}, \ldots, y_{n}\right)$ with adding the gradient of a function $f$ which depends on $\bar{y}$

$$
\Phi(x, y)=\left(\left(\phi^{*}\right)^{-1}(y) x+d f(\bar{y}), \phi(y)\right) .
$$

Using the action of the group $G_{H}$ on the Lagrangian germs we obtain the following result.

Theorem 3. A generic pair $(H, L ; p)$ such that $H$ is not transversal to $L$ at $p$ is symplectically equivalent to the pair $\left(H_{0}, L_{0} ; 0\right)$ obtained in Lemma 2 , where

$$
S(y)= \pm y_{1}^{k}+\sum_{i=2}^{k-2} y_{i} y_{1}^{k-i}+\left(g\left(y_{2}, \ldots, y_{k-2}\right) \pm \sum_{i=k-1}^{n} y_{i}^{2}\right) y_{1},
$$

provided $k=\operatorname{dim}_{R} \mathcal{E}_{y_{1}} / \Delta\left(S\left(y_{1}, 0\right)\right)+1 \leq n+2$, where $g \in \mathbf{m}_{y_{2}, \ldots, y_{k-2}}^{2} \backslash \mathbf{m}_{y_{2}, \ldots, y_{k-2}}^{3}$, and $\mathbf{m}_{y_{2}, \ldots, y_{k-2}}$ denotes the maximal ideal of the ring of smooth function-germs $\mathcal{E}_{y_{2}, \ldots, y_{k-2}}$.

Proof. $S$ is a deformation of the function $R \ni y_{1} \mapsto S\left(y_{1}, 0\right) \in R$ which satisfies (1). Therefore $S$ is equivalent to the pull-back of the universal deformation of $\pm y_{1}^{k}([3,8])$ :

$$
S(y)= \pm y_{1}^{k}+\sum_{i=2}^{k} \phi_{i}\left(y_{2}, \ldots, y_{n}\right) y_{1}^{k-i},
$$

where $\phi_{k-1} \in \mathbf{m}^{2}$. By the symplectomorphism

$$
\Phi(x, y)=\left(x, y-\operatorname{grad}\left(\phi_{k}\right)\right)
$$

we reduce $S$ to the form

$$
S(y)= \pm y_{1}^{k}+\sum_{i=2}^{k-1} \phi_{i}\left(y_{2}, \ldots, y_{n}\right) y_{1}^{k-i} .
$$

We may assume that $\left(\phi_{2}, \ldots, \phi_{k-2}\right)$ is a submersion and

$$
\phi_{k-1}\left(0, y_{k-1}, \ldots, y_{n}\right) \in \mathbf{m}_{y_{k-1}, \ldots, y_{n}}^{2} \backslash \mathbf{m}_{y_{k-1}, \ldots, y_{n}}^{3},
$$

because $(H, L ; 0)$ is generic. Therefore $S$ is equivalent to the germ

$$
S(y)= \pm y_{1}^{k}+\sum_{i=2}^{k-2} y_{i} y_{1}^{k-i}+\phi\left(y_{2}, \ldots, y_{n}\right) y_{1},
$$

where $\phi$ is deformation of a Morse function-germ $\phi\left(0, y_{k-1}, \ldots, y_{n}\right)$. Therefore by a lifting of a diffeomorphism of the form $\Psi(y)=\left(y_{1}, \ldots, y_{k-2}, \psi\left(y_{2}, \ldots, y_{n}\right)\right)$, we obtain $(2)$.

EXAMPLE 1 . Let $n=3$, then we have

$$
\begin{aligned}
& S\left(y_{1}, y_{2}, y_{3}\right)=y_{1}^{3}+y_{2}^{2} y_{1} \quad \text { (simple), } \\
& S\left(y_{1}, y_{2}, y_{3}\right)= \pm y_{1}^{4}+y_{2} y_{1}^{2}+\left(g\left(y_{2}\right) \pm y_{3}^{2}\right) y_{1}, g \in \mathbf{m}^{2}, \\
& S\left(y_{1}, y_{2}, y_{3}\right)=y_{1}^{5}+y_{2} y_{1}^{3}+y_{3} y_{1}^{2}+g\left(y_{2}, y_{3}\right) y_{1}, g \in \mathbf{m}^{2} .
\end{aligned}
$$


COROLlary 4. If $k>n+2$ then the normal form of $S$ under natural genericity conditions may be written in the following way

$$
S= \pm y_{1}^{k}+y_{i_{1}} y_{1}^{k-j_{1}}+\ldots+y_{i_{l}} y_{1}^{k-j_{l}}+\phi_{s_{1}}(\bar{y}) y_{1}^{k-s_{1}}+\ldots+\phi_{s_{r}}(\bar{y}) y_{1}^{k-s_{r}}+\psi(\bar{y}) y_{1},
$$

where $\left\{i_{1}, \ldots, i_{l}\right\}=\{2, \ldots, n\},\left\{j_{1}, \ldots, j_{l}, s_{1}, \ldots, s_{r}\right\}=\{2, \ldots, k-2\}, \phi_{s_{n}} \in \mathbf{m}, \psi \in \mathbf{m}^{2}$.

Remark 1 . All simple cases for $n=2$ are classified by $A_{k}$-singularities

$$
A_{l-1}: S\left(y_{1}, y_{2}\right)=y_{1}^{3} \pm y_{2}^{l} y_{1}, l \geq 2 \text {. }
$$

3. Coisotropic pairs. Now we slightly generalize the notion of a pair $(H, L ; p)$ taking instead of a hypersurface $H$ any coisotropic submanifold, say $V$. In the generic transversal case we have

Proposition 5. Let $V^{2 n-k}$ be a coisotropic submanifold of $M$, let $L$ be a Lagrangian submanifold of $M$ and $p \in V \cap L$. If $L$ is transversal to $V$ at $p$, then the pair $(V, L ; p)$ is symplectically equivalent to

$\left(V_{0}, L_{0} ; 0\right)=\left(\left\{(x, y) \in R^{2 n}: x_{1}=\ldots=x_{k}=0\right\},\left\{(x, y) \in R^{2 n}: y_{1}=\ldots=y_{n}=0\right\} ; 0\right)$.

Proof. We use the same method as in the proof of Theorem 1.

Lemma 6. If $L$ is not transversal to $V$ at $p$ then the pair $(V, L ; p)$ is symplectically equivalent to

$$
\begin{aligned}
& \left(V_{0}, L_{0} ; 0\right)=\left(\left\{(x, y) \in R^{2 n}: x_{1}=\ldots=x_{k}=0\right\},\right. \\
& \left.\left\{(x, y) \in R^{2 n}: x_{i}=-\partial S / \partial y_{i}(y) \text { for } i=1, \ldots, n\right\} ; 0\right),
\end{aligned}
$$

where $S$ is a function-germ such that the rank at 0 of the derivative of the mapping

$$
R^{n} \ni y \mapsto\left(-\partial S / \partial y_{1}(y), \ldots,-\partial S / \partial y_{k}(y)\right)
$$

is not maximal.

Proof. If $L$ is transversal at 0 to the submanifold

$$
\left\{(x, y) \in R^{2 n}: y_{1}=\ldots=y_{k}=0\right\}
$$

then we can find the generating function $S$, which depends on $\left(x_{I}, y_{J}\right)$, where $I \cup J=$ $\{1,2, \ldots, n\}, I \cap J=\emptyset$, such that

$$
\{1, \ldots, k\} \subset J
$$

and the rank at 0 of the derivative of the mapping

$$
R^{n} \ni\left(x_{I}, y_{J}\right) \mapsto\left(-\partial S / \partial y_{1}\left(x_{I}, y_{J}\right), \ldots,-\partial S / \partial y_{k}\left(x_{I}, y_{J}\right)\right)
$$

is not maximal. Otherwise $L$ is generated by a function $F$ which depends on $\left(x_{I}, y_{J}\right)$ such that condition (3) is not satisfied. Let $I_{1}=I \cap\{1, \ldots, k\}$ and $I_{2}=I \backslash\{1, \ldots, k\}$. We can find $F$ such that the rank at 0 of the derivative of the mapping

$$
R^{\left|I_{1}\right|} \ni x_{I_{1}} \mapsto \partial F / \partial x_{I_{1}}\left(x_{I_{1}}, 0\right) \in R^{\left|I_{1}\right|}
$$

is 0. By a symplectomorphism

$$
\Phi\left(x, y_{I_{1}}, y_{I_{2}}, y_{J}\right)=\left(x, y_{I_{1}}-x_{I_{1}}, y_{I_{2}}, y_{J}\right)
$$


which preserves $V_{0}$, we get transversality of $L$ to $\left\{(x, y) \in R^{2 n}: y_{1}=\ldots=y_{k}=0\right\}$ at 0 . Finally we use a symplectomorphism

$$
\Psi\left(x_{J}, x_{I}, y_{J}, y_{I}\right)=\left(x_{J},-y_{I}, y_{J}, x_{I}\right),
$$

which completes the proof.

Now we use the group of germs of symplectomorphisms which preserve the fibration $(x, y) \mapsto y$ and the submanifold $V_{0}$. This approach leads to the classification of families of functions of $k$ variables.

THEOREM 7. If $V^{2 n-k}$ is not transversal to $L$ at $p$ then the generic pair $\left(V^{2 n-k}, L ; p\right)$ is symplectically equivalent to the pair

$$
\left(\left\{(x, y) \in R^{2 n}: x_{1}=\ldots=x_{k}=0\right\}, L_{0} ; 0\right),
$$

where $L_{0}$ is generated by the function

$$
S(y)=f\left(y_{1}, \ldots, y_{k}\right)+\sum_{i=1}^{\mu-l-1} \phi_{i}\left(y_{k+1}, \ldots, y_{n}\right) \rho_{i}\left(y_{1}, \ldots, y_{k}\right)+\sum_{i=1}^{l} \psi_{i}\left(y_{k+1}, \ldots, y_{n}\right) y_{i},
$$

$\mu=\operatorname{dim}_{R}\left(\mathcal{E}_{y_{1}, \ldots, y_{k}} /\left\langle\partial f / \partial y_{1}, \ldots, \partial f / \partial y_{k}\right\rangle\right)<\infty, \rho_{i} \in \mathbf{m}_{y_{1}, \ldots, y_{k}}^{2}, \rho_{1}, \ldots, \rho_{\mu-l-1}, y_{1}, \ldots, y_{l}$ generate $\mathbf{m}_{y_{1}, \ldots, y_{k}}$ modulo $\left\langle\partial f / \partial y_{1}, \ldots, \partial f / \partial y_{k}\right\rangle$ and

$$
\operatorname{rank} D_{0}\left(\psi_{1}, \ldots, \psi_{l}\right)<l \text {. }
$$

4. Equivalence of Lagrangian submanifolds in the presence of a generic even-dimensional submanifold. First we formulate the result on the normal form of a symplectic surface in $\left(M, \omega_{0}\right)$.

LEMMA 8. If $X^{2(n-k)}$ is a symplectic submanifold of $\left(M, \omega_{0}\right)$ then at each point $p \in X^{2(n-k)}$ the germ $\left(X^{2(n-k)} ; p\right)$ is symplectically equivalent to

$$
\left(X_{0} ; 0\right)=\left(\left\{(x, y) \in R^{2 n}: x_{i}=y_{i}=0 \text { for } i=1, \ldots, k\right\} ; 0\right) .
$$

Pro of. By induction using the Moser method and the procedure described in [4].

Let us consider a symplectic pair $\left(X^{2(n-k)}, L ; 0\right)$. First we consider the transversal case. Since $L$ is transversal to the submanifold

$$
\left\{(x, y) \in R^{2 n}: x_{1}=\ldots=x_{k}=0\right\}
$$

we can express $L$ by a generating function $S: R^{n} \ni x \mapsto S(x) \in R$. The transversality assumption is equivalent to the following condition

$$
\begin{aligned}
& \operatorname{rank} D_{0}\left(R^{n-k} \ni\left(x_{k+1}, \ldots, x_{n}\right) \mapsto\right. \\
& \left.\quad\left(\partial S / \partial x_{1}\left(0, x_{k+1}, \ldots, x_{n}\right), \ldots, \partial S / \partial x_{k}\left(0, x_{k+1}, \ldots, x_{n}\right)\right) \in R^{k}\right)=k .
\end{aligned}
$$

Now we restrict our problem to the case $k=1$.

THEOREM 9. If $L$ is transversal to $X^{2(n-1)}$ at $p$ then the symplectic pair $\left(X^{2(n-1)}, L ; p\right)$ is symplectically equivalent to

$$
\begin{aligned}
& \left(X_{0}, L_{0} ; 0\right)=\left(\left\{(x, y) \in R^{2 n}: x_{1}=y_{1}=0\right\},\right. \\
& \left.\quad\left\{(x, y) \in R^{2 n}: y_{1}=x_{2}, y_{2}=x_{1}, y_{i}=0 \text { for } i=3, \ldots, n\right\} ; 0\right) .
\end{aligned}
$$


Proof. By Lemma 8 and the transversality assumption we can represent $X^{2 n-2}$ in the form

$$
\left\{(x, y) \in R^{2 n}: x_{1}=y_{1}=0\right\}
$$

and we can generate $L$ by a function

$$
S: R^{n} \ni x \mapsto S(x) \in R, S \in \mathbf{m}^{2}
$$

which satisfies the following condition: there exists $j \in\{2, \ldots, n\}$ such that

$$
\partial^{2} S / \partial x_{j} \partial x_{1}(0) \neq 0 \text {. }
$$

Now we expand $S$ in the form

$$
S(x)=x_{1}^{2} h(x)+x_{1} g\left(x_{2}, \ldots, x_{n}\right)+f\left(x_{2}, \ldots, x_{n}\right) .
$$

By a symplectomorphism

$$
\Phi(x, y)=\left(x, y+\operatorname{grad}\left(x_{1}^{2} h(x)+f\left(x_{2}, \ldots, x_{n}\right)\right)\right),
$$

which preserves $X^{2 n-2}$, we transform $L$ and consequently $S$ to the form

$$
S(x)=x_{1} g_{1}\left(x_{2}, \ldots, x_{n}\right) .
$$

By condition (4) the mapping

$$
\phi: R^{n} \ni x \mapsto\left(x_{1}, g_{1}\left(x_{2}, \ldots, x_{n}\right), x_{3}, \ldots, x_{j-1}, x_{2}, x_{j+1}, \ldots, x_{n}\right) \in R^{n}
$$

is a diffeomorphism. Now we use the symplectomorphism $\phi^{\star}: R^{2 n} \rightarrow R^{2 n}$, which completes the proof.

THEOREM 10. If $L$ is not transversal to $X^{2(n-1)}$ at $p$ then the generic symplectic pair $\left(X^{2(n-1)}, L ; p\right)$ is symplectically equivalent to the following pair

$$
\begin{aligned}
& \left(\left\{(x, y) \in R^{2 n}: x_{1}=y_{1}=0\right\},\right. \\
& \left.\left\{(x, y) \in R^{2 n}: y_{1}= \pm x_{1} \pm x_{2}^{2} \pm \ldots \pm x_{n}^{2}, y_{2}= \pm 2 x_{1} x_{2}, \ldots, y_{n}= \pm 2 x_{1} x_{n}\right\} ; 0\right) .
\end{aligned}
$$

Proof. $L$ is transversal to

$$
\left\{(x, y) \in R^{2 n}: x_{1}=0\right\} \text { or }\left\{(x, y) \in R^{2 n}: y_{1}=0\right\} \text {. }
$$

Otherwise $T_{0} L \subset T_{0} X$, which is impossible, because $L$ is an $n$-dimensional Lagrangian submanifold and $X^{2(n-1)}$ is a $(2 n-2)$-dimensional symplectic manifold. We may assume that $L$ is transversal to $\left\{(x, y) \in R^{2 n}: x_{1}=0\right\}$ at 0 . Then $(X, L ; 0)$ is symplectically equivalent to

$$
\left(\left\{(x, y) \in R^{2 n}: x_{1}=y_{1}=0\right\},\left\{(x, y) \in R^{2 n}: y=\partial S / \partial x(x)\right\} ; 0\right),
$$

where $S$ does not satisfy condition (4). By genericity of $(X, L ; p)$ we get

$$
\partial^{2} S / \partial x_{1}^{2}(0) \neq 0 \text {. }
$$

$S$ is a deformation of a function $R \ni x_{1} \mapsto S\left(x_{1}, 0\right)$ on the manifold with boundary $\left\{x \in R^{n}: x_{1}=0\right\}([2])$. Therefore it has the form

$$
S(x)= \pm x_{1}^{2}+g\left(x_{2}, \ldots, x_{n}\right) x_{1},
$$

and $d g_{0}=0$. We may assume that $g$ is a Morse function, which completes the proof. 


\section{References}

[1] V. I. Arnol'd, Singularities in variational calculus, Current Problems in Mathematics, 22, Itogi Nauki i Tekhniki, Akad. Nauk SSSR, Vsesoyuz. Inst. Nauchn. i Tekhn. Inform., Moscow, 1983, 3-55 (Russian).

[2] V. I. Arnol'd, Critical points of functions on a manifold with boundary, simple Lie groups $B_{k}, C_{k}, F_{4}$ and singularities of evolutes, Uspekhi Mat. Nauk 33(5) (1978), 91-105.

[3] V. I. Arnol' d, S. M. Guseŭn-Zade and A. N. Varchenko, Singularities of Differentiable Maps, Vol. 1, Birkhäuser, Boston, 1985.

[4] W. Domitrz and S. Janeczko, Normal forms of symplectic structures on the stratified spaces, Colloq. Math. 68 (1995), 101-119.

[5] M. Golubitsky and D. Tischler, An example of moduli for singular symplectic forms, Invent. Math. 38 (1977), 219-225.

[6] S. Janeczko and A. Kowalczyk, On singularities in the degenerated symplectic geometry, Hokkaido Math. J. 19 (1990), 103-123.

[7] J. Martinet, Sur les singularités des formes differentielles, Ann. Inst. Fourier (Grenoble) 20 (1970), 95-178.

[8] J. Martinet, Singularities of Smooth Functions and Maps, Cambridge Univ. Press, Cambridge, 1982.

[9] R. Roussarie, Modèles locaux de champs et de formes, Astérisque 30 (1975), 1-181.

[10] V. Zakalyukin, The generalization of lagrangian triads, Uspekhi Mat. Nauk 41(4) (1986), 180 . 